ANNALES

POLONICI MATHEMATICI

$83.3(2004)$

\title{
Holomorphic line bundles on a domain of a two-dimensional Stein manifold
}

\author{
by Mакото AвE (Kumamoto)
}

\begin{abstract}
Let $D$ be an open subset of a two-dimensional Stein manifold $S$. Then $D$ is Stein if and only if every holomorphic line bundle $L$ on $D$ is the line bundle associated to some (not necessarily effective) Cartier divisor $\mathfrak{d}$ on $D$.
\end{abstract}

1. Introduction. It is well known that every holomorphic line bundle $L$ on a projective algebraic manifold $P$ is associated to some (not necessarily effective) Cartier divisor $\mathfrak{d}$ on $P$ (see Griffiths-Harris [5, p. 161]). A similar fact holds for reduced Stein spaces. For every holomorphic line bundle $L$ on a reduced Stein space $S$ there exists a holomorphic global section $s$ of $L$ such that $s$ does not vanish identically on any irreducible component of $S$. Therefore $L$ is the line bundle associated to the effective Cartier divisor $\operatorname{div}(s)$ on $S$ (see Gunning [6, pp. 120-122]).

On the other hand a holomorphic line bundle $L$ on the punctured didisk $X:=\Delta^{2} \backslash\{(0,0)\}$, which is not Stein, is holomorphically trivial if and only if $L$ is the line bundle associated to some Cartier divisor on $X$ (see Remark 1.4 of Ballico [1]).

In this paper we prove that an open subset $D$ of a two-dimensional Stein manifold $S$ is Stein if and only if every holomorphic line bundle $L$ on $D$ is the line bundle associated to some (not necessarily effective) Cartier divisor $\mathfrak{d}$ on $D$ (Theorem 3). By using the method of Kajiwara-Kazama [10] the proof is deduced from the above-mentioned property of the punctured didisk.

If $S$ is a Stein manifold of dimension more than two, then there can exist an open subset $D$ of $S$ such that $D$ is not Stein and every holomorphic line bundle $L$ on $D$ is the line bundle associated to some effective Cartier divisor $\mathfrak{d}$ on $D$.

2. Lemmas. Let $X$ be a reduced complex space. Let $\mathfrak{d}$ be a Cartier divisor on $X$ defined by a meromorphic Cousin-II distribution $\left\{\left(U_{i}, m_{i}\right)\right\}_{i \in I}$

2000 Mathematics Subject Classification: 32E10, 32L10, 32T05.

Key words and phrases: holomorphic line bundle, Cartier divisor, Stein manifold. 
on $X$. We denote by $[\mathfrak{d}]$ the holomorphic line bundle on $X$ defined by the cochain $\left\{m_{i} / m_{j}\right\} \in Z^{1}\left(\left\{U_{i}\right\}_{i \in I}, \mathscr{O}^{*}\right)$. We say that $[\mathfrak{d}]$ is the holomorphic line bundle associated to $\mathfrak{d}$.

Let $\Delta:=\{t \in \mathbb{C}|| t \mid<1\}$. A holomorphic line bundle $L$ on the punctured didisk $X:=\Delta^{2} \backslash\{(0,0)\}$ is holomorphically trivial if and only if there exists a Cartier divisor $\mathfrak{d}$ on $X$ such that $L=[\mathfrak{d}]$ (see Remark 1.4 of Ballico [1]). More generally we have the following lemma.

Lemma 1. Let $S$ be a two-dimensional Stein manifold with $H^{2}(S, \mathbb{Z})$ $=0$. Let $A$ be a non-empty discrete subset of $S$. Let $L$ be a holomorphic line bundle on the open set $S \backslash A$. Then $L$ is holomorphically trivial if and only if there exists a Cartier divisor $\mathfrak{d}$ on $S \backslash A$ such that $L=[\mathfrak{d}]$.

Proof. Assume that there exists a Cartier divisor $\mathfrak{d}$ on $D:=S \backslash A$ such that $L=[\mathfrak{d}]$. Since $D$ is Cousin-II (see Theorem 8.25 of Hitotumatu [7, p. 174]), $L=[\mathfrak{d}]$ is holomorphically trivial. The converse is clear.

Let $S$ be a reduced complex space and $D$ an open subset of $S$. Let $p$ be a boundary point of $D$ in $S$. Then we say that $D$ is locally Stein at $p$ if there exists a neighborhood $U$ of $p$ in $S$ such that $U \cap D$ is Stein. By using the method of the proof of Lemma 11 of Kajiwara-Kazama [10] we prove the following lemma.

Lemma 2. Let $S$ be a purely two-dimensional reduced Stein space and $D$ an open subset of $S$. Assume that for every holomorphic line bundle $L$ on $D$ there exists a Cartier divisor $\mathfrak{d}$ on $D$ such that $L=[\mathfrak{d}]$. Then $D$ is locally Stein at every point $p \in \partial D \backslash \operatorname{Sing}(S)$.

Proof. Assume that there exists a point $p \in \partial D \backslash \operatorname{Sing}(S)$ such that $D$ is not locally Stein at $p$. Since $S$ is Stein, there exist a holomorphic map $\psi: S \rightarrow \mathbb{C}^{2}$ and a neighborhood $W$ of $p$ such that $W$ is non-singular, $\psi(W)$ is an open subset of $\mathbb{C}^{2}$ and $\left.\psi\right|_{W}: W \rightarrow \psi(W)$ is biholomorphic (see Grauert-Remmert [4, p. 151]). Take a Stein open subset $V$ of $\mathbb{C}^{2}$ such that $\psi(p) \in V \Subset \psi(W)$. Then $U:=\psi^{-1}(V) \cap W$ is a Stein neighborhood of $p$ and $\psi(U)=V$. Since $D$ is not locally Stein at $p$, the open set $\psi(D \cap U)$ is not Stein. By Lemma 1 of Kajiwara-Kazama [10] (see also the proof of Lemma 11 of [10]) there exist $H, P, \varepsilon, \varphi$ and $\left(b_{1}, b_{2}\right)$ with the following properties:

$$
\begin{aligned}
& H=\left\{\left(w_{1}, w_{2}\right) \in \mathbb{C}^{2}|| w_{1}|<1,| w_{2} \mid<1\right\} \\
& \cup\left\{\left(w_{1}, w_{2}\right) \in \mathbb{C}^{2}|1-2 \varepsilon<| w_{1}|<1+2 \varepsilon,| w_{2} \mid<1+2 \varepsilon\right\}, \\
& P=\left\{\left(w_{1}, w_{2}\right) \in \mathbb{C}^{2}|| w_{1}|<1+2 \varepsilon,| w_{2} \mid<1+2 \varepsilon\right\}, \quad 0<\varepsilon<1 / 2, \\
& \varphi: \mathbb{C}^{2} \rightarrow \mathbb{C}^{2} \text { is a biholomorphic map, } \quad \varphi(H) \subset \psi(D \cap U), \\
&\left|b_{1}\right| \leq 1-2 \varepsilon, \quad\left|b_{2}\right|=1, \quad \varphi\left(b_{1}, b_{2}\right) \in \partial(\psi(D \cap U)) .
\end{aligned}
$$


Let $\theta=\left(\theta_{1}, \theta_{2}\right):=\varphi^{-1} \circ \psi: S \rightarrow \mathbb{C}^{2}$. Let $T:=\left\{\left|\theta_{1}\right|<1+2 \varepsilon\right\}, T_{0}:=\left\{\left|\theta_{2}\right|<\right.$ $1+2 \varepsilon\} \cap T \cap U$ and $T_{1}:=\left\{\left|\theta_{2}\right|>1+\varepsilon\right\} \cup(T \backslash \bar{U})$. Then $T$ is a Stein open subset of $S$ and $\left\{T_{0}, T_{1}\right\}$ is an open covering of $T$. The function $1 /\left(\theta_{2}-b_{2}\right)$ is holomorphic on $T_{0} \cap T_{1}$. Since $H^{1}\left(\left\{T_{0}, T_{1}\right\}, \mathscr{O}\right)=0$, there exist $v_{i} \in \mathscr{O}\left(T_{i}\right)$, $i=1,2$, such that $1 /\left(\theta_{2}-b_{2}\right)=v_{1}-v_{0}$ on $T_{0} \cap T_{1}$. We define a meromorphic function $v$ on $T$ by the equalities $v=v_{0}+1 /\left(\theta_{2}-b_{2}\right)$ on $T_{0}$ and $v=v_{1}$ on $T_{1}$. Then $v$ is holomorphic on $\left(\left\{\theta_{2} \neq b_{2}\right\} \cap T_{0}\right) \cup T_{1}$. Let $D_{1}:=\left\{\theta_{1} \neq b_{1}\right\} \cap D$ and $D_{2}:=\left(\left(\left\{\theta_{2} \neq b_{2}\right\} \cap T\right) \cup(T \backslash \bar{U})\right) \cap D$. Then $\left\{D_{1}, D_{2}\right\}$ is an open covering of $D$ and the function $v /\left(\theta_{1}-b_{1}\right)$ is holomorphic on $D_{1} \cap D_{2}$. By assumption there exist invertible meromorphic functions $g_{i}$ on $D_{i}, i=1,2$, such that $\exp \left(v /\left(\theta_{1}-b_{1}\right)\right)=g_{1} / g_{2}$ on $D_{1} \cap D_{2}$. The function $g_{1}^{\prime}:=\exp \left(-v_{0} /\left(\theta_{1}-b_{1}\right)\right) g_{1}$ is meromorphic on $T_{0} \cap D_{1}$ and $\exp \left(1 /\left(\left(\theta_{1}-b_{1}\right)\left(\theta_{2}-b_{2}\right)\right)\right)=g_{1}^{\prime} / g_{2}$ on $T_{0} \cap D_{1} \cap D_{2}$. Let $H_{i}:=\left\{\left(w_{1}, w_{2}\right) \in H \mid w_{i} \neq b_{i}\right\}$ and $P_{i}:=\left\{\left(w_{1}, w_{2}\right) \in\right.$ $\left.P \mid w_{i} \neq b_{i}\right\}$ for $i=1,2$. Since $P$ is the envelope of holomorphy of $H$, the open set $P_{i}$ is the envelope of holomorphy of $H_{i}$ for each $i=1,2$ by Satz 7 of Grauert-Remmert [3] (see Theorem 2.5.9 of Jarnicki-Pflug [8, p. 182]). Since $H_{i} \subset \theta(U)$ and $\theta^{-1}\left(H_{i}\right) \cap U \subset T_{0} \cap D_{i}$ for $i=1$, 2, the function $f_{1}:=g_{1}^{\prime} \circ\left(\left.\theta\right|_{U}\right)^{-1}$ is meromorphic on $H_{1}$ and $f_{2}:=g_{2} \circ\left(\left.\theta\right|_{U}\right)^{-1}$ is meromorphic on $\mathrm{H}_{2}$. By Proposition 3 of Kajiwara-Sakai [11] there exists a meromorphic function $\tilde{f}_{i}$ on $P_{i}$ such that $\tilde{f}_{i}=f_{i}$ on $H_{i}$ for each $i=1,2$. Since $f_{i}$ is invertible on $H_{i}, \tilde{f}_{i}$ is also invertible on $P_{i}$ by the theorem of identity. We have $\exp \left(1 /\left(\left(w_{1}-b_{1}\right)\left(w_{2}-b_{2}\right)\right)\right)=\tilde{f}_{1} / \tilde{f}_{2}$ on $P_{1} \cap P_{2}$. This contradicts Lemma 1 because the function $\exp \left(1 /\left(\left(w_{1}-b_{1}\right)\left(w_{2}-b_{2}\right)\right)\right) \in \mathscr{O}^{*}\left(P_{1} \cap P_{2}\right)$ defines a non-trivial holomorphic line bundle on $P_{1} \cup P_{2}=P \backslash\left\{\left(b_{1}, b_{2}\right)\right\}$ (see Lemma 1 of Kajiwara [9] or Serre [12, p. 372]).

3. Theorem. We have the following theorem which characterizes a Stein open subset of a two-dimensional Stein manifold.

THEOREM 3. Let $S$ be a two-dimensional Stein manifold and $D$ an open subset of $S$. Then the following four conditions are equivalent.

(1) $D$ is Stein.

(2) For every holomorphic line bundle $L$ on $D$ there exists an effective Cartier divisor $\mathfrak{d}$ on $D$ such that $L=[\mathfrak{d}]$.

(3) For every holomorphic line bundle $L$ on $D$ there exists a Cartier divisor $\mathfrak{d}$ on $D$ such that $L=[\mathfrak{d}]$.

(4) The image of the natural homomorphism $H^{1}\left(D, \mathscr{O}^{*}\right) \rightarrow H^{1}\left(D, \mathscr{M}^{*}\right)$ vanishes.

Proof. $(3) \Rightarrow(1)$. By Lemma 2 the open set $D$ is locally Stein at every boundary point $p$ of $D$ in $S$. It follows that $D$ is Stein by the theorem of Docquier-Grauert [2]. 
$(1) \Rightarrow(2)$. Every holomorphic line bundle $L$ on an arbitrary reduced Stein space is associated to some Cartier divisor [ $\mathfrak{d}]$. For the proof of this fact we refer to Gunning [6, pp. 120-122].

$(2) \Rightarrow(3) \Leftrightarrow(4)$. Clear.

For an open subset $D$ of a Stein manifold $S$ such that $\operatorname{dim} S \geq 3$ the theorem above does not hold. As an example, we take a non-empty analytic subset $A$ of $S$ such that $\operatorname{codim} A \geq 3$. The open subset $D:=S \backslash A$ of $S$ is not Stein. Let $L$ be an arbitrary holomorphic line bundle on $D$. There exists a holomorphic line bundle $\tilde{L}$ on $S$ such that $\left.\tilde{L}\right|_{D}=L$ (see Shiffman [13, p. 340]). Since $S$ is Stein, there exists an effective divisor $\tilde{\mathfrak{d}}$ on $S$ such that $\tilde{L}=[\tilde{\mathfrak{d}}]$. Then we have $L=\left[\left.\tilde{\mathfrak{d}}\right|_{D}\right]$ and condition (2) of Theorem 3 is satisfied.

\section{References}

[1] E. Ballico, Holomorphic vector bundles on $\mathbb{C}^{2} \backslash\{0\}$, Israel J. Math. 128 (2002), 197-204.

[2] F. Docquier und H. Grauert, Levisches Problem und Rungescher Satz für Teilgebiete Steinscher Mannigfaltigkeiten, Math. Ann. 140 (1960), 94-123.

[3] H. Grauert und R. Remmert, Konvexität in der komplexen Analysis. Nicht-holomorph-konvexe Holomorphiegebiete und Anwendungen auf die Abbildungstheorie, Comment. Math. Helv. 31 (1956), 152-183.

[4] -, -, Theory of Stein Spaces, Grundlehren Math. Wiss. 236, Springer, Berlin, 1979.

[5] P. Griffiths and J. Harris, Principles of Algebraic Geometry, Wiley, New York, 1978.

[6] R. C. Gunning, Introduction to Holomorphic Functions of Several Variables, Vol. 3, Wadsworth, Belmont, CA, 1990.

[7] S. Hitotumatu, Theory of Analytic Functions of Several Variables, Baifûkan, Tokyo, 1960 (in Japanese).

[8] M. Jarnicki and P. Pflug, Extension of Holomorphic Functions, de Gruyter, Berlin, 2000 .

[9] J. Kajiwara, On Thullen's example of a Cousin-II domain, Sci. Rep. Kanazawa Univ. 9 (1964), 1-8.

[10] J. Kajiwara and H. Kazama, Two dimensional complex manifold with vanishing cohomology set, Math. Ann. 204 (1973), 1-12.

[11] J. Kajiwara and E. Sakai, Generalization of Levi-Oka's theorem concerning meromorphic functions, Nagoya Math. J. 29 (1967), 75-84.

[12] J.-P. Serre, Prolongement de faisceaux analytiques cohérents, Ann. Inst. Fourier (Grenoble) 16 (1966), no. 1, 363-374.

[13] B. Shiffman, Extension of positive line bundles and meromorphic maps, Invent. Math. 15 (1972), 332-347.

School of Health Sciences

Kumamoto University

Kumamoto 862-0976, Japan

E-mail: mabe@hs.kumamoto-u.ac.jp 